\title{
Clichés balzaciens : une esthétique de l'ambiva- lence
}

\author{
Maria GAL \\ Université de Genève
}

\begin{abstract}
Cet article propose une réflexion sur le lieu commun ; entendu, conformément à Aristote, comme mode d'expression didactique privilégié. À partir d'une analyse du cliché balzacien, il s'agira de déterminer quelles sont les composantes formelles de ce «mode d'expression » et quels en sont les mécanismes. Ce qui permettra, dans un deuxième temps, de répondre aux questions suivantes : comment le cliché «parle-t-il » aux « masses »? Et dans quel but ? Enfin, l'article élargira la perspective, et visera à établir des parallèles entre les clichés balzaciens et d'autres formes, antérieures, de mise en narration didactique des images : les paraboles.
\end{abstract}

Keywords : Balzac, lieu commun, métaphore, didactique, préjugé

En cherchant à caractériser le lieu commun, Antoine Compagnon avait pointé « son ambivalence » : « [à] la fois il fait penser et il empêche de penser $»^{\mathrm{I}}$. Cette ambivalence, Compagnon la considère du point de vue de l'histoire littéraire, s'interrogeant sur la valeur que l'on accorde ou non au topos, selon qu'on le considère « digne de la tradition » ou « manquant d'originalité ». Question qui renvoie à la querelle sans cesse recommencée des anciens et des modernes.

Mais en amont de son aspect purement esthétique, le lieu commun, dans son ambivalence, pose une autre question : celle de sa légitimité. Jusqu'à quel point la généralisation qu'il opère est-elle juste, et à partir de quel moment devient-elle excessive, discriminatrice ou tout simplement bête ? Comment, par exemple, distingue-t-on un «type » d'un «stéréotype » ? Car à la base de l'un et de l'autre, il y a une opération de généralisation, un souci d'ordre, de classification. Le « lieu commun » est, littéralement, le lieu à partir duquel plusieurs personnes parviennent à s'entendre sur un seul et même objet.

D'un point de vue sociologique, selon Pascal Durand, «pas de société sans stéréotype, pas de lien social sans lieux communs par où le maintenir noué, pas d'existence collective sans rapports de conformité réciproque entre les représentations mentales des individus $»^{2}$. Mais ces stéréotypes fédérateurs sont aussi le talon d'Achille d'une communauté : c'est par là qu'elle est vulnérable, manipulable.

I Antoine Compagnon, «Théorie du lieu commun », Cahiers de l'Association internationale des études françaises, 49, 1997, p. 23.

2 Pascal Durand, «Lieu commun, cliché, stéréotype. Généalogie des formations figées », in 
Pour Ruth Amossy33, la distinction, même si elle est difficile à établir, tient au fait que le « mauvais » lieu commun vise (volontairement ou non) à faire passer pour vrai quelque chose qui ne l'est pas forcément; à présenter comme fondée une assertion qui ne l'est pas ; à prétendre absolue une idée arbitraire. La limite est d'autant plus ténue qu'elle est mobile : ce qui était vrai par le passé peut ne plus l'être aujourd'hui ; ce qui est vrai pour certains ne l'est pas pour d'autres. D'où la difficulté de vouloir louer ou blâmer le lieu commun. Et celle, aussi, d'en faire un usage adéquat.

En reliant ces deux dimensions du lieu commun, esthétique et sociale, on aboutit à une troisième question, essentielle : celle de la place tenue par le lieu commun dans la communauté littéraire. Au sens restreint, la " communauté littéraire » désigne les producteurs de littérature. À cette échelle, le rapport à la vox populi dont est issu le lieu commun consiste plutôt en une mise à distance - mise à distance qui entérine la valeur intrinsèque de la littérature, la légitime en tant que forme d'expression particulière. Au sens large, la «communauté littéraire » désigne non seulement les producteurs de littérature, mais aussi ses consommateurs ; c'est-à-dire, la société dans son ensemble. Et à cette échelle, la question n'est pas de savoir comment l'auteur se démarque de la société productrice de lieux communs, mais au contraire - comment il entre en contact avec elle. Comment parle-t-on à quelqu'un de ses préjugés ? Comment dénonce-t-on une stéréotypie jugée abusive ? De quel droit le fait-on ? À partir de quelle position? En quels termes? C'est à ces dernières questions qu'est consacré cet article. Le problème est si vaste, si complexe, que je n'envisage de proposer ici que quelques pistes de réflexion. Je le ferai à partir de l'œuvre de Balzac, si féconde en lieux communs.

Avant d'aborder l'auteur, je souhaiterais effectuer un bref retour aux origines de la notion. Il est intéressant de remarquer que les problèmes soulevés par le lieu commun sont à la base même de sa théorisation. Et que dans cette théorisation, loin d'être perçus comme des problèmes ils constituent les arguments de départ. Le topos grec est l'ancêtre du lieu commun. Dans ses Topiques, Aristote l'intègre à une « méthode pour argumenter sur tout problème à partir de prémisses probables $»^{4}$. Probables, c'est-à-dire non vraies. Voilà qui, d'office, confirme le point précédemment évoqué : les lieux communs ne relèvent pas d'une vérité absolue, mais relative. Voyons à présent quel rôle jouent ces " prémisses probables » (c'est-à-dire les topoï) au sein du système aristotélicien. En tant que méthode du probable,

Conférences de l'école doctorale de science politique, éd. Lucien Sfez, Paris, Publications de la Sorbonne, 2004, pp. 45-46.

3 Ruth Amossy, "Types ou stéréotypes ? Les "Physiologies" et la littérature industrielle ", Romantisme, 1989, 64, pp. II3-I23.

4 Aristote, Les Topiques, I, I, Iooar8. 
la dialectique relève d'un champ propre - distinct de la science du vrai ; celui de la contestation d'opinions qui semblent évidentes à beaucoup. Mais pour arriver à l'argument qui met l'adversaire en déroute, il faut pouvoir délimiter des points d'ancrage communs. De tels ancrages sont les topoi ${ }^{5}$.

Sans ces topiques, et cela malgré leur ambivalence, on ne pourrait pas passer d'une opinion, partagée au départ par les interlocuteurs, à son dépassement.

Après Les Topiques, consultons Les Seconds Analytiques. Voici une précision de ce qu'Aristote entend par « probable » : "l'opinion est chose instable [...]. Jamais on ne pense avoir une simple opinion quand on pense que la chose ne peut être autrement : on pense alors qu'on a la science. Mais quand on pense que rien n'empêche que la chose soit autrement - alors, on a une opinion $»^{6}$. Et nous touchons là à l'essentiel : pour réfuter une opinion admise (un lieu commun, un cliché, un préjugé), il faut montrer que la chose (le lieu commun) est opinion - non science.

Considérons enfin La Rhétorique, c'est-à-dire la concrétisation du programme :

Il faut parler par maximes pour aller contre les clichés [...]. Les maximes sont d'un grand secours pour l'orateur, fût-ce pour la simple raison que les auditeurs sont des gens primaires : ils sont contents si l'orateur, formulant des choses de manière générale, rencontre les opinions qui sont les leurs [...]. Ainsi l'orateur doit pressentir quel est son auditoire et quels sont ses préjugés, pour ensuite, alors, s'exprimer sur un plan général7 7 .

Ensuite, Aristote précise que « les fables conviennent le mieux au discours devant le peuple ». Enfin, il donne des indications pratiques : « ce qui fait l'excellence de la diction, c'est la clarté. Le discours ne doit être ni plat ni pompeux $»^{8}$. Il faut toutefois

donner au langage un air insolite [...]. Le mot courant, le mot propre et la métaphore sont les seuls moyens utiles au discours. Une preuve : c'est d'eux, et d'eux seuls que tout le monde se sert, puisque tout le monde les utilise dans la conversation. Dès lors, il est clair que si le poète la manie bien, la diction aura, sans y paraître, un air insolite, tout en gardant sa clarté9 .

5 Michel Meyer, «Introduction », Revue internationale de philosophie, 270, 20I4, p. 39I.

6 Aristote, Les Seconds Analytiques, I, 33, 89a.

7 Id., La Rhétorique, II, 2I, 1395a-b.

8 Ibid., III, 2, I404b.

9 Ibid. 
Nous voyons que le lieu commun, à son origine, est complet : pas de rupture fondamentale entre l'opinion publique (le lieu commun qui en est issu), la rhétorique, et la poétique. Ni entre un réservoir de phrases toutes faites et son actualisation par l'orateur ou le poète ; c'est-à-dire entre une prescription théorique et un usage social.

Passons à présent de la théorisation aristotélicienne du lieu commun à l'œuvre de Balzac. Nous verrons sous peu que le lien entre l'une et l'autre est bien plus étroit qu'il n'y paraît au premier abord.

Quelle est l'ambition, à la fois philosophique et sociale, de l'auteur de $\mathrm{La}$ Comédie humaine? S'autorisant de son statut d'auteur de fiction, Balzac ambitionne de décrire tout (l'ensemble de la société française du XIx ${ }^{e}$ siècle) ; sous tous ses aspects (César Birotteau, par exemple, est l'histoire d'une faillite qui sera montrée du point de vue du failli, de sa femme, de sa fille, du prétendant de sa fille, de son banquier, de son entourage, etc.) ; pour tout lecteur ; en même temps.

Pourquoi pour tout lecteur? Balzac publie ses romans en feuilletons, et veut intéresser le lectorat le plus vaste possible ${ }^{\mathrm{IO}}$ : féminin et masculin, érudit ou non. Si le livre coûte cher, le feuilleton est plus abordable. Le romancier, largement impliqué dans le journalisme et l'édition, avait cherché à promouvoir ce mode de publication, notamment par le système des abonnements : afin, d'une part, de permettre à la librairie de sortir de la crise dans laquelle elle se trouvait, et d'autre part d'élargir le lectorat tout en le fidélisant ${ }^{\mathrm{II}}$.

La question qui se pose d'emblée, avec un tel projet, est celle de la réalisation : «comment [demande Balzac dans son Avant-propos] plaire à la fois au poète, au philosophe et aux masses qui veulent la poésie et la philosophie sous de saisissantes images? $\gg^{12}$. Et nous revenons là au propos initial : comment parler avec " clarté » - disait Aristote - au " peuple », comment l'amener à la généralisation philosophique sans tomber dans le cliché et, si possible, en le détournant de ses préjugés? Aristote, nous l'avons vu, préconise de recourir à la logique des « lieux communs » : s'exprimer en un discours clair et métaphorique ; s'adresser à l'opinion courante dans son propre langage.

Io Sur la question du rapport de Balzac aux différentes catégories de lecteurs, voir Jérôme David, Balzac, une éthique de la description, Paris, Champion, 2010.

II Voir Honoré de Balzac, «De l'état actuel de la librairie », in Euvres diverses, 2 vol., éd. PierreGeorges Castex, Paris, Gallimard, « Bibliothèque de la Pléiade », I990-I996, t. II, pp. 662-670 ; et « Acte préliminaire. Exposant les bases, le but et les moyens de l'entreprise dite la société d'abonnement général », ibid., pp. 853-863.

I2 Id., "Avant-Propos ", in La Comédie humaine, I2 vol., éd. Pierre-Georges Castex, Paris, Gallimard, «Bibliothèque de la Pléiade», I976-I98I, t. I, p. 4. 
C'est précisément ainsi que procède Balzac : c'est sous la forme de «saisissantes images » qu'il ambitionne de présenter la philosophie de ses romans. Et c'est cette même forme qui lui sera reprochée par la critique littéraire, de Sainte-Beuve à Barthes et au-delà. D'un point de vue stylistique, en effet, ces images dérangent. Proust les évoque avec un mélange d'attrait et de répulsion :

dans Balzac [...] coexistent, non digérés [...] tous les éléments d'un style à venir qui n'existe pas. Le style ne suggère pas, il explique. Il explique d'ailleurs à l'aide des images les plus saisissantes, qui font comprendre ce qu'il veut dire comme on le fait comprendre dans la conversation si on a une conversation géniale ${ }^{13}$.

Suit un exemple : «M. du Châtelet était comme ces melons qui de verts deviennent jaunes en une nuit $»^{14}$. Curtius en parle presque dans les mêmes termes :

Nous nous trouvons en présence de clichés vieux comme le monde, de fleurs de rhétorique qui continuent à s'étioler dans les basses eaux de la littérature [...]. Des formules abstruses, des métaphores excessives, des effets comiques douteux restent ça et là non digérés, comme une matière brute [...]. Seulement [et cette dernière remarque de Curtius me parait essentielle], Balzac les emploie d'une façon si fréquente et si caractéristique qu'il faut bien attribuer à cette habitude une signification particulière ${ }^{15}$.

Nous savons que Balzac est capable de produire autre chose (certains passages stylistiquement "purs » en témoignent), mais il sature délibérément ses textes de clichés douteux. Pourquoi ? Puis ces clichés, comment les comprendre ? On ne peut pas - et on ne doit pas - les restreindre à un sens symbolique. Ils s'ancrent solidement dans le sens premier. Proust déplorait déjà cette prégnance du sens littéral chez Balzac:

Tandis que souvent chez les écrivains le titre est plus ou moins un symbole, une image qu'il faut prendre dans un sens plus poétique [...], avec Balzac, c'est plutôt le contraire. La lecture de cet admirable livre qui s'appelle Les Illusions perdues restreint et matérialise plutôt ce beau titre. Il signifie que Lucien de Rubempré venant à Paris s'est rendu compte que Mme de Bargeton

I3 Marcel Proust, «Sainte-Beuve et Balzac », in Contre Sainte-Beuve, Paris, Gallimard, I954, p. 201.

I4 Ibid., p. 203.

I5 Ernst-Robert Curtius, Balzac, trad. Henri Jourdan, Paris, Grasset, 1933, pp. 345-346. 
était ridicule et provinciale, que les journalistes étaient fourbes, que la vie était difficile [...]. Chaque titre doit ainsi être pris au pied de la lettre ${ }^{16}$.

Prendre les titres au pied de la lettre ; et les images aussi. Reste encore à savoir comment cela fonctionne. Poser des clichés ne revient pas encore à les relativiser; et ce n'est pas l'un ou l'autre, pris séparément, qui produit du sens : pour arriver à cette philosophie du cliché, il faut les mettre en rapport les uns avec les autres.

L'exemple sur lequel je m'appuierai pour élucider cette question est tiré des premières pages d'Ursule Mirouët. Le roman s'ouvre sur la description d'un personnage, Minoret-Levrault. Cette description constitue un véritable florilège de clichés. Et les remarques de Curtius que je viens d'évoquer ont justement été suscitées par ce passage :

À l'aspect de Minoret-Levrault un artiste aurait quitté le site [de Nemours] pour croquer ce bourgeois, tant il était original à force d'être commun. Réunissez toutes les conditions de la brute, vous obtenez Caliban qui certes, est une grande chose. Là où la Forme domine, le Sentiment disparaît. Le maître de poste, preuve vivante de cet axiome, présentait une de ces physionomies où le penseur aperçoit difficilement trace d'âme sous la violente carnation que produit un brutal développement de la chair. Sa casquette en drap bleu [...] moulait une tête dont les fortes dimensions prouvaient que la science de Gall n'a pas encore abordé le chapitre des exceptions. Les cheveux gris et comme lustrés qui débordaient la casquette vous eussent démontré que la chevelure blanchit par d'autres causes que par les fatigues d'esprit ou par les chagrins. De chaque côté de la tête, on voyait de larges oreilles presque cicatrisées sur les bords par les érosions d'un sang trop abondant qui semblait prêt à jaillir au moindre effort [...]. Les yeux gris, agiles, enfoncés [...] ressemblaient aux yeux des Kalmouks venus en I8I5 [...]. Le nez, déprimé depuis sa racine, se relevait brusquement en pied de marmite [...]. [Sa physionomie avait le caractère] de la puissance stupide que les sculpteurs impriment à leurs cariatides. Minoret-Levrault ressemblait à ces statues, à cette différence près qu'elles supportent un édifice et qu'il avait assez à faire de se soutenir lui-même. Vous rencontrerez beaucoup de ces Atlas sans monde. Le buste de cet homme était un bloc ; vous eussiez dit d'un taureau relevé sur ses deux jambes de derrière. Les bras vigoureux se terminaient par des mains épaisses et dures [...] qui pouvaient et savaient manier le fouet, les guides, la fourche, et auxquelles aucun postillon ne se jouait. L'énorme ventre de ce géant était supporté par des cuisses grosses comme le corps d'un adulte et par des pieds d'éléphant. La colère devait être rare chez cet homme, mais terrible, apoplectique alors qu'elle éclatait. Quoique violent et incapable de réflexion, cet homme n'avait rien fait qui justifiât les sinistres

I6 Proust, «Sainte-Beuve et Balzac», art. cit., p. 200. 
promesses de sa physionomie. À qui tremblait devant ce géant, ses postillons disaient : - Oh ! il n'est pas méchant !7

Afin de mieux comprendre les enjeux du texte, voici un bref résumé du début de l'intrigue : Minoret-Levrault dont on vient de lire la description est le neveu du docteur Minoret. Le docteur Minoret a adopté une jeune fille, Ursule. Il mourra sous peu, et léguera sa fortune à Ursule ; Minoret-Levrault s'arrangera pour faire disparaître le testament et voler la succession de la pupille.

Considérons pour commencer une interprétation désormais classique du passage, empruntée à Max Andréoli :

Peut-être aperçoit-on mal dans le passage quels liens s'établissent entre Minoret-Levrault, Caliban, un axiome sur la Forme et le Sentiment [...]. Et pourtant, ils existent [...]. L'impression d'extravagance ressentie par certains lecteurs tient au fait qu'ils négligent, par respect excessif des normes du "bon goût", de replacer le fragment dans l'ensemble de La Comédie humaine ${ }^{18}$.

Jusque là, rien à redire. Mais dès que s'amorce l'analyse, la perspective change. Quittant soudain le domaine du sens commun, le critique s'élance dans celui de la pure abstraction :

La Forme, l'un des aspects de la Matière, s'oppose au Sentiment, l'un des avatars de l'Esprit, de même que les comparaisons qui les caractérisent. Forme et Sentiment se rattachent à l'idée d'un antagonisme brutal, animal, de la Chair avec la Pensée, donc à un axe majeur de l'œuvre ${ }^{19}$.

Ce à quoi, en définitive, aboutit l'analyse d'Andréoli n'est pas la relativisation de l'axiome, mais son entérinement.

La dichotomie est-elle réellement si tranchée, d'une symétrie aussi parfaite ? Reprenons le texte. Minoret-Levrault est " la preuve vivante » de l'axiome selon lequel « là où la Forme domine, le Sentiment disparaît ». Balzac part d'une opinion générale, communément admise, puisqu'un axiome est une "vérité ou assertion admise par tous sans discussion " Balzac, de fait, part d'un lieu commun. On s'attend à ce que la description maintienne deux perspectives : une vision spirituelle, élevée - celle de la

I7 Balzac, Ursule Mirouët, in La Comédie humaine, op. cit., t. III, pp. 770-77I. Dorénavant, les références à ce roman seront indiquées par le sigle UM et placées entre parenthèses dans le texte. I8 Max Andréoli, Le Système balzacien. Essai de description synchronique, Lille, Presses de l'Université Lille III, I984, p. 830.

I9 Ibid., p. 83I.

20 Cnrtl, http://www.cnrtl.fr/definition/axiome. 
«Pensée »-, versus une vision matérielle, triviale - celle de la « Chair». Mais l'intention du texte est autre. Minoret-Levrault est placé à un point de vue stratégique : «Si le but de la poésie est de mettre les idées au point précis où tout le monde peut les voir et les sentir [affirme Lucien dans Les Illusions perdues], le poète doit incessamment parcourir l'échelle des intelligences humaines, afin de les satisfaire toutes $»^{21}$.

Qu'entend Balzac par « toutes »? Reprenons le texte pour répondre : l'artiste ; "vous », lecteur, qui que vous soyez; " vous » en particulier, lecteur de Shakespeare ; le penseur; le physiognomoniste ; le phrénologue ; le spécialiste en canitie ; le médecin ; celui qui aurait vu des Kalmouks dans les troupes alliées qui envahirent Paris en 1815 ; la ménagère, qui sait à quoi ressemble un pied de marmite ; l'historien de l'art ; l'amateur d'allégories ; le postillon; tout spectateur qui « tremblait » à la vue du géant.

Tout cela ne concerne encore que la «Forme » du maitre de poste. «Forme » qu'il faut mettre en relation avec le «Sentiment». Mais d'abord, qu'est-ce que le «sentiment»? C'est la faculté de s'élever par l'âme et par l'esprit. C'est aussi celle d'éprouver des passions néfastes : la colère, la haine, la jalousie. C'est encore la capacité de réfléchir, l'intelligence. Enfin, c'est la sensation la plus élémentaire, l'instinct ${ }^{22}$. Tout cela, supposément, est compris dans le maître de poste.

«Le penseur » ou l'artiste gardent en tête l'antagonisme conceptuel : en face de la "Forme » ils ne perçoivent pas « trace d'âme » - c'est-à-dire, pas trace du « Sentiment » qui permet de «s'élever par l'âme ». Le physiognomoniste, lui, se trouve confronté à un cas étrange : les «fortes dimensions » de la tête ne correspondent pas au schéma attendu - et dans ce cas, "Sentiment » renvoie aux capacités intellectuelles. Le médecin décèle des anomalies : le « sang [est] trop abondant », et présente la menace d'un débordement; dans ce sens, le « Sentiment » est la manifestation des humeurs, des passions. Puis la «puissance stupide» des cariatides, est, chez Minoret-Levrault, larvée. Cette latence de la « Forme » se mue en celle du « Sentiment » obscurément perçu par les postillons : ses «mains pouvaient manier le fouet »- mais envers qui ? Et sous quelle impulsion ? Celle de la colère ? De l'instinct ? La puissance de la " Forme " pourrait-elle, en dépit de l'assertion, se révéler dans le « Sentiment » ? Et ce dernier, que désigne-t-il en dernière instance ?

Si le rapport de la «Forme » et du «Sentiment » constitue une vérité générale, nous voyons qu'il peut être investi, suivant les perspectives, de significations différentes. Les métamorphoses progressives semblent indi-

2I Balzac, Les Illusions perdues, in La Comédie humaine, op. cit., t. V, p. 207.

22 Cnrtl, http://www.cnrtl.fr/definition/sentiment. 
quer que les apparences sont trompeuses; que l'axiome peut prendre les allures du paradoxe, s'avérer aussi vrai d'un certain point de vue, que faux d'un autre.

Considérons un autre exemple :

On a toujours assez d'esprit pour concevoir une lésion d'intérêts. L'intérêt constitue l'esprit du paysan aussi bien que celui du diplomate, et sur ce terrain le plus niais en apparence serait peut-être le plus fort. Aussi ce terrible raisonnement : "Si la petite Ursule a le pouvoir de jeter son protecteur dans le giron de l'Église, elle aura bien celui de se faire donner sa succession ", éclatait-il en lettres de feu dans l'intelligence du plus obtus des héritiers (UM, 802).

Voilà que le «Sentiment » apparaît dans le sens d'« esprit » qui lui était initialement dénié. L'axiome est-il réfuté pour autant ? N'est-il pas plutôt modulé ? Le maître de poste a " assez d'esprit pour concevoir » une idée : c'est-à-dire qu'il en a peu. C'est un être «niais ». En ce sens, il est dépourvu d'intelligence. Mais si l'on change de perspective et que l'on se place sur le «terrain » de «l'intérêt », le même maître de poste, comparé au « diplomate », « serait peut-être le plus fort ». Ici, au sein même de l'axiome, la conception de l'intelligence est double. Dans la perspective généralisante de la maxime (" on a toujours »), elle est absente. Mais dans la perspective, particulière, des intérêts financiers, elle est effective.

On voit que la question n'est plus de savoir si l'axiome est vrai ou faux, si l'antinomie est universelle - mais de voir tout ce qu'elle est susceptible de signifier. Encore un exemple : le clerc de Nemours promet à Minoret-Levrault de faire « crever [Ursule] de chagrin » (UM, 94I). En échange de ce service, le maître de poste l'établira notaire. Ursule est mourante, et le clerc vient réclamer son dû. Minoret-Levrault s'en débarrasse à moindre frais. Voici l'extrait :

Le premier clerc, en voyant l'imbécile et colossal Machiavel de la rue des Bourgeois dans un accès de fièvre seigneuriale, lui jeta pour adieux un : « Au revoir ! » et un regard qui eussent fait trembler tout autre qu'un niais parvenu, regardant du haut d'une terrasse les jardins et les magnifiques toits d'un château bâti dans le style à la mode sous Louis XIII (UM, 949).

Hors contexte, l'image est « extravagante » : comment un « Machiavel » peut-il être « niais » ? Et pourquoi doit-il nécessairement être « imbécile et colossal »? La « Forme » domine dans ce passage. Et le « Sentiment » ? Il est absent, si l'on considère qu'un « vrai » Machiavel aurait dû se méfier du clerc. Mais si l'on se rappelle qu'en intérêts, « le plus niais en apparence serait peut-être le plus fort »? Et en sachant que le vol est impuni, qu'Ursule 
est mourante, que Minoret-Levrault est passé de la rue des Bourgeois au château, et que le clerc a été berné ; le machiavélisme n'est-il pas remarquable? Enfin, ne perçoit-on pas un effet d'annonce ambigu ? Ne voit-on pas en Minoret-Levrault, simultanément, le Machiavel le plus adroit, enrichi par sa haine et son " esprit paysan » - et le plus vulnérable, sabotant lui-même ses succès par sa bêtise?

«Forme » et « Sentiment » sont encore appelés à subir un grand nombre de métamorphoses. Le dénouement, dans un nouveau florilège de clichés, exploite l'axiome jusqu'au bout de ses possibilités. Venons-en à présent à ce dernier passage. Il livre l'ultime portrait de Minoret-Levrault. Pressé par le remords, le maître de poste a restitué la fortune dérobée à Ursule. Atterré par la mort de son fils, travaillé par le repentir, il est méconnaissable :

Il est devenu l'homme le plus charitable, le plus pieux de Nemours [...]. Si vous avez remarqué sur le bord des chemins, dans les pays où on étête le chêne, quelque vieil arbre blanchi et comme foudroyé, poussant encore des jets, les flancs ouverts et implorant la hache, vous aurez une idée du vieux maître de poste, en cheveux blancs, cassé, maigre, dans qui les anciens du pays ne retrouvent rien de l'imbécile heureux que vous avez vu attendant son fils au commencement de cette histoire; il ne prend plus son tabac de la même manière, il porte quelque chose de plus que son corps. Enfin, on sent en toute chose que le doigt de Dieu s'est appesanti sur cette figure pour en faire un exemple terrible (UM, 986).

Les effets de symétrie entre la description qui ouvre le récit et celle qui le clôt sont nombreux : les « jets » qui poussent encore sur le vieux chêne font écho aux « regains » qui débordaient des « charmantes prairies ». De «grand et gros », Minoret-Levrault est devenu « cassé et maigre ». L' « imbécile heureux » de l'incipit est devenu « l'homme le plus charitable » de Nemours, et celui qui « attend[ait] son fils au commencement de cette histoire » l'a désormais perdu. Enfin, " l'Atlas sans monde » qui « avait assez à faire à se soutenir lui-même » " porte quelque chose de plus que son corps ». L'inversion est parfaitement calculée : c'est finalement là où le «Sentiment » domine que la «Forme » disparaît. Les thèses classiques de l'analyse balzacienne se trouvent confirmées : la puissance destructrice de la passion, ses ravages sur le corps ; la suprématie de l'Esprit sur la Matière. Et, comme chez de nombreux personnages balzaciens, une fin de vie marquée par le repentir et l'expiation. Au niveau de la structure du texte, on peut abonder dans le sens des conclusions de Barthes :

On connaît la loi de la solidarité du lisible : tout se tient, tout doit se tenir le mieux possible [...] : c'est en somme le calcul qui fait le plein de cette littérature : la dissémination n'y est pas l'éparpillement perdu des sens vers 
l'infini du langage, mais une simple suspension - provisoire - d'éléments affinitaires, déjà aimantés, avant qu'ils ne soient convoqués et n'accourent pour se ranger économiquement dans le même paquet ${ }^{23}$.

Dans cet ultime cliché, tous les éléments " se range[nt] » sémantiquement en deux « paquet[s] » : le registre du « Sentiment » immatériel, symbole de la rédemption chrétienne, triomphe définitivement de celui de la chair et de toute "Forme » concrète. "Quelque chose de plus que son corps " renvoie à un élément autre que physique, au spirituel. De même pour « sentir ", qui évoque ici le fait d'éprouver un sentiment. «En toute chose » ne signifie pas un objet précis, mais l'existence en général. Quant au « doigt de Dieu », Balzac utilise ici l'image dans sa forme et sa fonction allégoriques : c'est la représentation matérielle d'une puissance qui ne saurait pas l'être, mais qui, pour être comprise, revêt une forme humaine. Enfin, par le choix des termes dont il se sert pour conférer au récit son exemplarité (Minoret-Levrault est la « figure » dont « le doigt de Dieu » a « fai[t] un terrible exemple »), il l'inscrit dans la veine moralisante et didactique des exempla ${ }^{24}$.

Et pourtant, l'impression qui s'en dégage est tout autre : la «pureté » de l'image succombe sous le poids des «fleur[s] de rhétorique » qui la soustendent ; fleurs généreusement semées par Balzac. Qu'en résulte-t-il ? L'émergence de deux dimensions différentes au sein de l'image. Dans un même mot, parallèlement à l'idée de spiritualité exprimée par le sens figuré, se développe celle de la matière, véhiculée par le sens littéral. Minoret-Levrault " porte quelque chose de plus que son corps ", c'est-à-dire qu'il est écrasé par un poids. C'est aussi en «toute chose » que l' " on sent » ce poids, c'est-à-dire, physiquement. Que sent-on ? Que « le doigt de Dieu s'est appesanti »; l'image est éloquente. Et sur quoi s'est appesanti ce doigt ? Sur la «figure » du maître de poste ; c'est-à-dire sur toute sa personne. Enfin, quel est le but ? D'en "faire un exemple » - c'est-à-dire de modeler les traits d'un individu à l'image d'une exemplarité. Ce réengagement de la matière mine le principe même de dissociation du concret et de l'abstrait ; tant au niveau du fond («Forme » et «Sentiment » ne devant manifestement pas être pensés en termes d'exclusion mutuelle) que de la forme (le littéral et le figuré requérant une lecture conjonctive). Une maladresse serait-elle aussi systématique? Je ne le pense pas. Pourquoi, dès lors, induire une telle ambivalence?

23 Roland Barthes, S/Z, Paris, Seuil, 1970, pp. 172-I73.

24 Les exempla sont de courtes fables très répandues au Moyen Âge : à partir d'un «fait divers », les clercs développaient un discours moralisant et imagé, destiné à édifier leurs ouailles. Le héros de la fable, pour s'être adonné à quelque vice, subissait toutes sortes de péripéties fâcheuses, et finissait généralement mal. Voir Aaron Gurevitch, La Culture populaire au Moyen Âge. Simplices et docti, trad. Elena Balzamo, Paris, Aubier, 1996. 
C'est que cette dernière est constitutive de l'image du « doigt de Dieu ", reprise et réactualisée par Balzac. L'expression se trouve employée dans l'Ancien Testament à l'occasion des plaies d'Égypte (Exode $8: 15$ ); et dans le Nouveau à propos d'un exorcisme effectué par le Christ (Luc II : 20). Dans ces deux épisodes, la question est de savoir si un miracle (invasion de moustiques dans le premier cas, guérison d'un muet dans le second) est le fait de la volonté divine. Ce qui est donc interrogé, c'est le rapport entre une cause spirituelle et un effet physique. Ce rapport est exprimé par le « doigt » de Dieu. Au sens littéral, le « doigt » désigne un élément matériel : une partie de l'anatomie, constituée de chair et d'os. Au figuré, cette partie anatomique est instrumentalisée : elle ne vaut que par sa capacité à réaliser une intention (" montrer du doigt », « toucher du doigt », « ne pas lever le petit doigt », etc.). Dans le « doigt » figurent donc les deux éléments du rapport : le matériel et le spirituel. Cette ambivalence est redoublée par le texte; dans un cas comme dans l'autre, le « doigt » est médiatisé par une figure anthropomorphique: Aaron, le Christ. Tous deux sont des êtres en chair et en os ; mais ils sont en même temps des esprits soumis à la volonté divine. La formulation ne cherche manifestement pas à trancher la question de ce rapport, mais à la développer ${ }^{25}$. Sa logique vise l'approfondissement des formes (pluralité des images, pluralité des niveaux de signification) non la définition $d u$ sens: l'effectivité du «Verbe » peut se manifester par un « doigt », par un homme, par un bâton, par le fils de Dieu. Elle peut transformer le sable en moustiques, faire sortir le mutisme d'un corps.

Du déluge à la résurrection, de ses manifestations les plus spectaculaires aux plus intériorisées, le « Verbe » sera inlassablement dramatisé, exprimé par une infinité de "saisissantes images " plus ou moins narrativisées (les paraboles), lesquelles reproduisent dans leur structure formelle, par la coprésence également significative des sens littéral et figuré, l'ambivalence du rapport entre le spirituel et le matériel. La médiatisation humaine, déjà présente dans l'Ancien Testament, se trouve radicalisée par le mythe christique dans le Nouveau. Dieu n'envoyant plus ni moustiques ni sauterelles, c'est à l'échelle de l'être humain que s'établit le rapport : libérée de l'intervention directe du créateur, autonomisée, la scène sur laquelle se déroule le combat entre le «bien » et le « mal » est désormais l'homme ${ }^{26}$. Or c'est précisément

25 Dans les deux épisodes, la question reste délibérément ouverte : l'origine divine de l'invasion de moustiques n'est pas affirmée ; elle est déduite de l'incapacité des magiciens à la reproduire, et n'est formulée que par ces derniers. De même, dans l'exorcisme du muet, c'est sous forme d'hypothèse que le Christ énonce son argument : «si c'est par le doigt de Dieu que moi je chasse les démons... ». D'où la diversité des interprétations, des débats sur la supra-naturalité (ou non) des phénomènes cataclysmiques (notamment dans les exégèses rabbiniques), à l'assimilation augustinienne du « doigt » au Saint-Esprit.

26 Déplacement dont témoigne, par exemple, le succès de la Psychomachia de Prudence, fort en vogue au Moyen Âge. C'est pareillement à échelle humaine que sont entreprises les 
ici qu'il faut prendre garde à une erreur fort répandue. Elle consiste en l'assimilation simpliste de deux couples, présentés comme similairement antithétiques : l' « esprit»/le «corps »; le « bien»/le «mal». Nous venons de voir que le premier exprime non pas une opposition mais un rapport, caractérisé par une grande polymorphie. Le second désigne au contraire une forte polarité, à l'aune de laquelle sont évalués divers éléments. Le rapport de l'esprit et du corps, dans la pensée religieuse, peut également mener au « mal » ou au « bien ». C'est pour cette raison que le lien entre chair et esprit, dans la théologie chrétienne, a toujours davantage été pensé en termes d'étroite corrélation (problématique, mais par cela même cruciale), que d'exclusion mutuelle ${ }^{27}$, cette dernière étant le fait de courants gnostiques et manichéens, taxés d'hérésie. D'où, dans le domaine savant de l'exégèse, l'intérêt pour les « saisissantes images » des Écritures, qui présentent le spirituel sous forme de chair. Ces images, il s'est agi de les rationnaliser; tâche à laquelle se sont attelés les Pères de l'Église en développant l'allégorèse. Cette méthode herméneutique consiste à considérer les paraboles au sens figuré et au sens littéral; à réfléchir au rapport entre ces deux niveaux de $\operatorname{sens}^{28}$, quitte à ensuite faire primer tel sens sur tel autre ${ }^{29}$ (sans, toutefois, que le sens littéral puisse jamais être totalement évacué : en tant que sens « historique», attestant de la véridicité du mythe christique, il est essentiel).

À partir de la fin du XII siècle, l'essor de la scolastique, la pensée de Thomas d'Aquin, la redécouverte d'Aristote ont contribué à transformer l'allégorèse en une «théologie scientifique ». La rationalisation de la pluralité des sens en est progressivement venue à viser la limitation ; le but, désormais,

«saintes » missions des croisés ; lesquels ne se définissaient plus comme le « doigt " mais comme le «bras » de Dieu sur terre.

27 Jean-Claude Schmitt, Le Corps, les rites, les rêves, le temps. Essais d'anthropologie médiévale, Paris, Gallimard, 20oI.

28 Voir, par exemple, chez Augustin : «Qu'on dise, par exemple, que les hommes saints [...] sont ceux dont l'Église [...] propose la vie et les mœurs comme exemples aux infidèles [...], pour les arracher à leurs vaines superstitions, et se les incorporer $[. .]:$. comment se fait-il qu'ainsi exprimée cette vérité frappe moins agréablement l'auditeur, que si on la lui découvre figurée dans ce passage du Cantique des cantiques, où on adresse à l'Église cette louange [...] : "Tes dents sont comme un troupeau de brebis tondues qui montent du lavoir, et qui portent un double fruit sans qu'il y en ait de stérile parmi elles ?" Y découvrons-nous une vérité différente de celle qui nous était présentée en termes clairs et sans figure ? Et cependant je ne sais pourquoi je contemple avec plus de charme les saints quand je les considère comme les dents de l'Église, arrachant les hommes au joug de l'erreur, brisant la pureté de leurs cœurs, les broyant et les triturant en quelque sorte pour les lui incorporer » (De la doctrine chrétienne, livre 2, chapitre VI, 7). À noter que le panégyrique des dents (comme celui du palais d'ailleurs, et pour les mêmes raisons) est devenu, dans la littérature mystique, un lieu commun fort répandu.

29 Je n'en évoque que deux pour la clarté du propos; mais ils sont généralement au nombre de quatre (historique ; allégorique ; tropologique ; anagogique). Voir Henri de Lubac, Exégèse médiévale. Les quatre sens de l'Écriture, Paris, Aubier, 1959-I964. 
étant de combattre les détracteurs de l'Église sur le terrain de la raison, en démontrant "scientifiquement » la légitimité du dogme. Or c'est paradoxalement en cherchant à entériner le sens orthodoxe des Écritures que cette théologie a œuvré à son ébranlement. Mettant à nu les formes du mythe chrétien et leurs mécanismes, "l'exégèse biblique, avec ses quatre niveaux de sens, concourait à la désacralisation du Livre sacré. Les clercs réfléchissaient-ils [...] sur les sacrements et la notion de miracle ? C'était réduire d'autant les possibilités d'action du surnaturel et le pouvoir de Dieu sur terre $»^{30}$. Nous voyons que soumise à l'analyse, l'image, par son ambivalence, résiste à toute tentative de réduction - quitte à faire exploser le moule dans lequel on cherche à la faire rentrer. En tant que forme narrative, elle vise la dramatisation des rapports, non leur catégorisation.

Dans sa version vulgarisée (les exempla précédemment évoqués), la pensée religieuse présentée aux « masses » n'échappait pas, elle non plus, à l'ambivalence :

En lisant ces ouvrages édifiants, on est toujours frappé par un paradoxe : il y a une sorte de contradiction entre le sujet et la façon de le traiter [...]. Argumenter la nécessité de fuir le péché et de résister aux tentations [...], appeler à la pénitence et au repentir [...] : tels sont le plus souvent les thèmes des courts récits (exempla) [...]. Or tous ces pieux objectifs doivent être atteints ici-bas [...] par des hommes qui, pour la plupart, ne pensent qu'à servir leurs propres intérêts grossièrement matériels et égoïstes ${ }^{31}$.

Ni le « doigt de Dieu » ni la posture du maître de poste, conséquemment, ne peuvent ni ne doivent être pensés dans la perspective de l'« antagonisme brutal », prétendument " axe majeur de l'œuvre», proposé par Andréoli. Le «Sentiment » s'exprime par la « Forme », dont il ne fait que réaliser les possibilités ; et inversement. Tous deux s'avèrent étroitement liés dans une chaîne de rapports causaux ; chaîne dont Balzac démontre la complexité, chaque maillon, suivant la perspective choisie, renvoyant potentiellement à des conséquences, des prémisses différentes. Que conclure, par exemple, de la canitie de Minoret-Levrault ? Le remords en a fait un homme « en cheveux blancs ». Mais au début du récit, ne lisions-nous pas que «la chevelure blanchit par d'autres causes que par les fatigues d'esprit ou par les chagrins » - et n'était-il pas à supposer que c'est la convoitise ou l'appétit qui avaient prématurément vieilli le maître de poste ? Attrape-t-on des cheveux blancs par excès de désir ou de culpabilité ? Peut-être - par les deux?

30 Schmitt, Le Corps, les rites, les rêves, le temps, op. cit., p. 52.

3I Gurevitch, La Culture populaire au Moyen Âge, op. cit., p. 313. 
Revenons, pour finir, à Curtius : « Que Balzac [...] ait possédé jusqu’à un degré peu commun le don de communiquer, d'évoquer, d'animer, de mettre les âmes en contact, personne ne le niera $»^{32}$. C'est ce que nous venons de voir. "Animer ", c'est mettre une généralité axiomatique à l'épreuve du particulier. Viser non pas l'univocité du vrai, mais la pluralité des possibles : l'axiome en puissance. Et cette opération, Balzac l'effectue à grand renfort de clichés. "Pires ", esthétiquement parlant, les uns que les autres ; mais efficaces, et accessibles. Qu'en conclure? Qu'avec Balzac, il faut se méfier des apparences; ou du moins, penser à les interroger.

32 Curtius, Balzac, op. cit., p. 34I. 